\title{
Cosmic Microwave Background anisotropies beyond the third peak
}

\author{
N. Aghanim ${ }^{1,3}$, P. G. Castro ${ }^{2}$, A. Melchiorri ${ }^{2}$, and J. Silk ${ }^{2}$ \\ 1 IAS-CNRS, Université Paris Sud, Bât. 121, 91405 Orsay Cedex, France \\ 2 Denys Wilkinson Building, University of Oxford, Keble Road, Oxford, OX 3RH, UK \\ 3 IAP-CNRS, 98 bis Boulevard Arago, 75014 Paris, France
}

Received 11 March 2002 / Accepted 11 July 2002

\begin{abstract}
Forthcoming Cosmic Microwave Background (CMB) experiments will allow us to accurately investigate the power spectrum at very small scales $(\ell>1000)$. We predict the level of the primary anisotropies, given the actual CMB measurements. The secondary anisotropies generated after matter-radiation decoupling contribute additional power in the tail of the CMB power spectrum. Together with the primary anisotropies, we compute the predicted power spectra for three dominant secondary effects induced by photon scattering. We predict these secondary contributions in flat cosmological models for parameters in agreement (to $2 \sigma$ ) with the values allowed by current parameter estimates.
\end{abstract}

Key words. cosmology: cosmic microwave background

\section{Introduction}

The last several years have been an exciting period for observational cosmology, particularly in the field of the Cosmic Microwave Background (CMB) research. With recent $\mathrm{CMB}$ balloon-borne and ground-based experiments such as TOCO (Miller et al. 1999), BOOMERanG (de Bernardis et al. 2000; Mauskopf et al. 2000; Netterfield et al. 2001), MAXIMA (Hanany et al. 2000; Lee et al. 2001; Stompor et al. 2001), and DASI (Halverson et al. 2001), we are entering a new era of precision cosmology that enables us to use the CMB anisotropy measurements to constrain the cosmological parameters and the underlying theoretical models. From all of these experiments, a firm detection of the first peak in the CMB anisotropy angular power spectrum has now been obtained. Moreover, in the framework of adiabatic cold dark matter (CDM) models, the position, amplitude and width of the measured peak provide strong evidence for the inflationary predictions of a low curvature (flat) universe and a scale-invariant primordial spectrum (Dodelson \& Knox 2000; Melchiorri et al. 2000). Furthermore, the latest results from BOOMERanG (Netterfield et al. 2001) and DASI (Halverson et al. 2001) point to the presence of second and third peaks, confirming the theoretical prediction of acoustic oscillations in the primeval plasma and shedding new light on various cosmological and inflationary parameters (de Bernardis et al. 2002; Pryke et al. 2001; Wang et al. 2001). The locations and amplitudes of three acoustic peaks and two dips in the last releases of the CMB data have been determined

Send offprint requests to: $\mathrm{N}$. Aghanim, e-mail: Nabila.Aghanim@ias.u-psud.fr in a model-independent way in recent work (see e.g. Durrer et al. 2001). In general, the location and amplitude of the first acoustic peak are determined at more than $3 \sigma$ confidence. The next two peaks and dips are determined at a confidence level above $1 \sigma$ but below $2 \sigma$. It is however worth mentioning the work of Miller et al. (2002), which finds that the second peak is a $2 \sigma$ detection and that the third peak is not detected to any reasonable significance.

The next generation of CMB experiments will be even more powerful as they are designed to achieve, down to arcminute scales, accurate measurements of both the temperature anisotropies and polarised emission of the CMB. Through these data, we expect multiple oscillations in the spectrum to be unambiguously detected and, thanks to the polarisation measurements, this should enable us to remove some of the degeneracies that are still affecting parameter estimation.

However, the present CMB data, as we will see in one of the following sections, already constrain the shape of the angular power spectrum of primary anisotropies with good accuracy. The present poor determination of some of the cosmological and astrophysical parameters is more related to intrinsic degeneracies (different models leading to the same spectrum) rather than to the precision of the actual CMB measurements. Given this uncertainty, we can forecast in a rather reliable way the level of primary anisotropies on arcminute and subarcminute scales, where the effect of secondary anisotropies (i.e. produced well after matter-radiation decoupling) should start to dominate and hence significantly affect the CMB measurements. The secondary anisotropies can be generated due to photon interactions with the matter potential wells for 
example in the Rees-Sciama effect (Rees \& Sciama 1986), lensing (Seljak 1996) and the "butterfly" effect (Birkinshaw \& Gull 1983). The other secondary anisotropies are induced by the interaction of CMB photons with free electrons such as in the Sunyaev-Zel'dovich (SZ) effect (Sunyaev \& Zel'dovich 1980), the Ostriker-Vishniac (OV) effect (Ostriker \& Vishniac 1986; Vishniac 1987), and from the effects of early inhomogeneous reionisation (Aghanim et al. 1996; Gruzinov \& Hu 1998; Knox et al. 1998).

In the present work, we first forecast the level of primary anisotropies on arcminute and sub-arcminute scales by comparing the recent CMB data with a template of cosmological models (Sect. 2). We then quantify, in Sect. 3, the contribution of the secondary scattering effects that are likely the dominant contributions at small scales. The contributions are computed for a set of cosmological parameters allowed by the present data (within $2 \sigma$ error bars). The corresponding power spectra are presented together with our results in Sect. 4. Our discussion and conclusions are given in Sect. 5.

\section{Forecasting primary anisotropies}

Our goal in this section is to forecast the level of primary anisotropies on very small scales $(\ell>1000)$, given the actual CMB measurements that span an interval in angular scales of $2<\ell<1000$. The first step in our procedure consists of building a template of cosmological models and of comparing each model with the CMB observations through a likelihood. The theoretical models of our template are computed using the publicly available cMBFAST program. They are defined by 6 parameters sampled as follows: $\omega_{\mathrm{cdm}}=\Omega_{\mathrm{cdm}} h^{2}=0.01, \ldots 0.40$ (step 0.01); $\omega_{\mathrm{b}}=\Omega_{\mathrm{b}} h^{2}=0.001, \ldots, 0.040\left(\right.$ step 0.001); $\Omega_{\Lambda}=$ $0.0, \ldots, 1.0$ (step 0.05) and $\Omega_{\mathrm{k}}$ such that $\Omega_{\mathrm{m}}=0.1, \ldots, 1.0$ (step 0.05). The value of the Hubble constant is not an independent parameter, since $h=\sqrt{\left(\omega_{\mathrm{cdm}}+\omega_{\mathrm{b}}\right) /\left(1-\Omega_{\mathrm{k}}-\Omega_{\Lambda}\right)}$. We vary the spectral index of the primordial density perturbations within the range $n_{\mathrm{s}}=0.60, \ldots, 1.40$ (step 0.02) and we rescale the amplitude of fluctuations by a pre-factor $C_{10}$, in units of $C_{10}^{\mathrm{COBE}}$, with $0.50<C_{10}<1.40$.

The models of the template are then compared with the recent BOOMERanG-98, DASI and MAXIMA-1 results. The power spectra from these experiments were estimated in 19,9 and 13 bins respectively, spanning the range $25 \leq \ell \leq 1150$. For the DASI and MAXIMA-I experiments, we use the publicly available correlation matrices and window functions. For the BOOMERanG experiment, we assign a flat space for the spectrum in each bin $\ell(\ell+1) C_{\ell} / 2 \pi=C_{\mathrm{B}}$; we approximate the signal $C_{\mathrm{B}}$ inside the bin to a Gaussian variable and we consider $\sim 10 \%$ correlations between the various bins. We consider $10 \%, 4 \%$ and $5 \%$ Gaussian distributed calibration errors (in $\mu K$ ) for the BOOMERanG-98, DASI and MAXIMA-1 experiments respectively and we included the beam uncertainties by the analytical marginalisation method presented in Bridle et al. (2001). We also include the COBE data using Lloyd Knox's RADPack packages. The likelihood for a given cosmological model is then defined by $-2 \ln \mathcal{L}=\left(C_{\mathrm{B}}^{\text {th }}-C_{\mathrm{B}}^{\text {ex }}\right) M_{\mathrm{BB}^{\prime}}\left(C_{\mathrm{B}^{\prime}}^{\text {th }}-C_{\mathrm{B}^{\prime}}^{\text {ex }}\right)$ where $M_{\mathrm{BB}}$, is the Gaussian curvature of the likelihood matrix at the peak. We then plot in the $\ell(\ell+1) C_{\ell}-\ell$ plane the enve- lope of all the models that are consistent at $95 \%$ c.l. with the CMB data (Fig. 2). This provides us with the predicted power spectrum of the primary anisotropies as deduced from the actual data. We consequently define above this band the region where, at $95 \%$ c.l., a contribution from primary anisotropies is unlikely to be present. In performing the envelope of all the models, we have neglected the correlations between different $\ell$ modes for a single model: this simple procedure is also the most conservative approach. Moreover, our computations rely on two main assumptions:

- The primary CMB can be described by one of the theoretical models in our template (i.e. we do not consider possible deviations such as scale-dependent spectral indexes, isocurvature primordial perturbations or topological defects);

- The present CMB data are not affected by unknown foregrounds or systematics.

The first assumption is a theoretical prior mainly supported by the good agreement between the theoretical template and the present CMB data. The same underlying model seems also to fit the shape of the matter power spectrum on small scales obtained, for example, from recent galaxy surveys. The second assumption is based on the numerous tests performed by the experimental teams to estimate the Galactic contamination in the so-called CMB channels (150 or $30 \mathrm{GHz}$ ). It also relies, in particular, on the point source extraction carried out by the DASI team. Moreover, the overall consistency of the CMB data from these experiments that have sampled various regions of the sky, at different frequencies and with different techniques (bolometers and radiometers) reinforces our confidence in this second assumption. In the following, we will generalise the standard concept of foregrounds to include the secondary anisotropies that cannot be spectrally disentangled from the primary anisotropies, and thus corrected for, by the present experiments, and we will investigate their possible contributions.

\section{Secondary contributions}

An increasingly large number of studies have been devoted to the CMB anisotropies in general, and to the secondary anisotropies in particular. We refer the reader to $\mathrm{Hu} \&$ Dodelson (2002) and White \& Cohn (2002). The latter paper gives a rather comprehensive guide to the literature on the topics related to the $\mathrm{CMB}$, including secondary anisotropies. In the present study, we concentrate on the dominant contributions due to secondary effects generated through interactions with electrons. The induced temperature anisotropies contribute to the total signal at different angular scales. Between a few arcminutes to a few tens of arcminutes, the SZ effect dominates, whereas at smaller angular scales typically down to arcminute scales the extra power essentially comes from the OV effect.

\subsection{Sunyaev-Zel'dovich effect}

The SZ effect (see Birkinshaw 1999 for a recent review) is a well-studied effect which designates the inverse Compton interaction of the CMB photons with the free electrons of the 
hot intra-cluster medium. This effect is usually "decomposed" into two parts: the so-called thermal SZ (TSZ), and the kinetic SZ (KSZ) effects. The TSZ amplitude is a measure of the pressure integral along the line of sight. It is directly linked to the intra-cluster gas properties (temperature $T_{\mathrm{e}}$ and optical depth $\tau$ ). It has a very peculiar spectral signature that translates into an excess of brightness at high frequencies $(850 \mu \mathrm{m})$ and a decrement at low frequencies $(2 \mathrm{~mm})$. For hot clusters, it can be modified to include relativistic corrections (see, e.g. Rephaeli 1995). The KSZ effect corresponds to a first-order Doppler effect when a galaxy cluster moves with respect to the CMB rest frame with a radial peculiar velocity $v_{\mathrm{r}}$. The KSZ anisotropies have the same spectral signature as the primary CMB anisotropies.

The SZ effect is one of the most important sources of secondary anisotropies. Due to its spectral signature, the TSZ can in principle be separated from the other components (in particular from the CMB primary anisotropies) by means of accurate multi-wavelength observations in the millimetre/submillimetre range (Hobson et al. 1998; Bouchet \& Gispert 1999; Snoussi et al. 2001). The only remaining contribution is therefore that associated with the KSZ anisotropies which cannot be separated from the primary fluctuations via multi-wavelength measurements. However, most of the present and future CMB experiments due to their lack of frequency coverage are unable to detect and subtract the TSZ anisotropies from the measurements. This contribution is thus likely to be present in the measured signal. In the present study, we investigate the SZ anisotropies using a semi-analytical model, the most recent version of which (assumptions and procedures) is described in detail in Aghanim et al. (2001). It is based on the computation of predicted cluster number counts from a Press-Schechter (Press \& Schechter 1974) mass function modified, according to Sheth \& Tormen (1999) and Wu (2000), to better fit the numerical predictions of the number of dark matter halos in the mass range $5 \times 10^{13}$ to $10^{16}$ solar masses. Each cluster is physically described by its intra-cluster gas temperature (assumed isothermal) and electron density distribution (following the well-known $\beta$-profile, $\beta=2 / 3$ ). Provided with these quantities and the cluster peculiar velocities, both TSZ and KSZ anisotropies are computed for the cluster population. The SZ contribution (power spectrum) is then computed via simulated maps of both KSZ and TSZ components.

Several techniques, based on analytic or semi-analytic computations and on numerical simulations, can be used to predict the SZ signal of a cluster population (as for example in Cooray 2000; da Silva et al. 2000; Komatsu et al. 2000; Refregier \& Teyssier 2000; Majumdar 2001; Springel et al. 2001 (and references therein)). These authors give different predictions, especially for the power spectrum. An illuminating comparison illustrating this point has been performed by Springel et al. (2001). The most noticeable result of the comparison is a scatter of about one order of magnitude in the SZ power spectra at all angular scales. This scatter is partially due to the differences between the different methods used for the computation, and to their intrinsic limitations. We note, for example, that the semi-analytic predictions apparently give somehow higher amplitudes than numerical simulations. As for the latter, they are

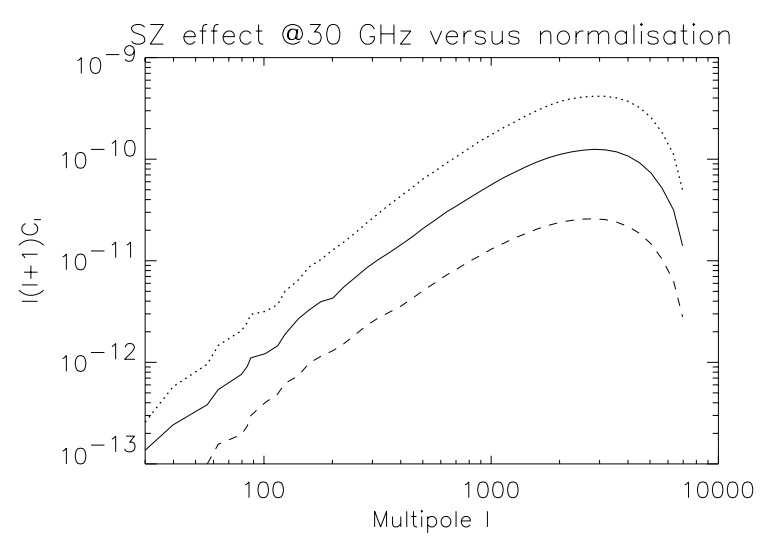

Fig. 1. The SZ power spectrum computed for the "concordance model" ( $h=0.71, \Omega_{\mathrm{m}}=0.3, \Omega_{\Lambda}=0.7$ ). The three curves represent the variation of the power spectrum with the normalisation factor $\sigma_{8}=\sigma_{8}^{0} \Omega_{\mathrm{m}}^{-0.47}$ (from Viana \& Liddle 1999). The dashed line is obtained for $\sigma_{8}^{0}=0.4$, the solid line for 0.5 and the dotted line for 0.6 .

limited by numerical resolution, which especially affects the results at small angular scales. In addition, the amplitude of the SZ contribution crucially depends on the physical assumptions entering in the computation. The observed variations in the predicted power spectra can be due on the one hand to the predicted number of structures and on the other hand to the physical description of the intra-cluster gas, and its evolution with redshift. In this context, one of the most influencial parameters for the number count computations is the normalisation factor $\sigma_{8}$ which is also rather uncertain. The amplitude of the SZ power spectrum can indeed vary by an order of magnitude with rather small variations of $\sigma_{8}$. This dependence is illustrated in Fig. 1 where we use the expression given in (Viana \& Liddle 1999): $\sigma_{8}=\sigma_{8}^{0} \Omega_{\mathrm{m}}^{-0.47}$ with $\sigma_{8}^{0}=0.4$ (dashed line), 0.5 (solid line) and 0.6 (dotted line), and $\Omega_{\mathrm{m}}=0.3$. It is therefore important to realise that due to our present limited understanding of the structure formation and evolution (especially for the gaseous part), the predicted SZ power spectra only provide an order of magnitude estimate of the effect rather than exact and precise amplitudes. In the following, we will illustrate the results using $\sigma_{8}^{0}=0.5$.

\subsection{Inhomogeneous reionisation}

It has become generally accepted that the universe underwent reionisation that was completed around a redshift $z_{i} \sim 6$. The evidence for reionisation relies on observations of the Gunn-Peterson (Gunn \& Peterson 1965) effect (both in hydrogen and helium) towards distant quasars (Becker et al. 2001). Several physical processes have been proposed to achieve the total reionisation of the universe. Among them, photoionisation by early luminous sources (stars, quasars, dwarf galaxies) has recently been the most favoured. Indeed, this leads to a rather simple and natural scenario in which the first luminous sources emit ionising photons that ionise hydrogen atoms in their vicinities. In this model, reionisation starts as an inhomogeneous process, and the total reionisation is reached when the ionised bubbles overlap (due to their increasing number 
and to their expansion). The most recent observations of very distant quasars $(z \sim 6)$ are now suggesting that we have reached the point where we are starting to observe the period of inhomogeneous reionisation (IHR) (Becker et al. 2001; Gnedin 2001). As pointed out by Aghanim et al. (1996), inhomogeneous reionisation can induce secondary anisotropies of the KSZ type, when the ionised bubbles move with respect to the CMB rest frame. The inhomogeneous, or patchy, reionisation has been during the few last years the subject of numerous recent studies. Among these, we cite for example Benson et al. (2001), Ciardi et al. (2001), Gnedin \& Jaffe (2001) and Bruscoli et al. (2002)

We estimate the possible contribution (in terms of the power spectrum) of the anisotropies due to the IHR by using the simple model proposed by Gruzinov \& Hu (1998), which we have generalised to a non-critical universe (open or flat with non-zero cosmological constant). This is an empirical model of the IHR based on three parameters: the redshift at which ionisation starts $z_{\mathrm{s}}$, its duration $\delta z$ and the typical size of the ionised bubble $R$. In our case, we add the constraint that reionisation is completed at $z_{\mathrm{i}}=6$, i.e. for a given $z_{\mathrm{s}}$ this fixes $\delta z$. In addition, we set $z_{\mathrm{s}}=10$ which is a reasonable assumption for the formation of the first objects, i.e. when ionisation starts. However, this simple model is limited by the fact that it gives a predicted power spectrum for a specified bubble size that for illustration we set to $10 \mathrm{Mpc}$. It therefore introduces an artificial cut-off at small angular scales. A smaller (larger) size, shifts the power spectrum to smaller (larger) angular scales and decreases (increases) the amplitude.

\subsection{Ostriker-Vishniac effect}

Along with the IHR effect produced during the first stages of reionisation and independently of any physical model to explain the sources of photoionisation, we have to take into account the well-studied Ostriker-Vishniac effect OV (Ostriker \& Vishniac 1986; Vishniac 1987; Hu et al. 1994; Dodelson \& Jubas 1995; Jaffe \& Kamionkowski 1998) which arises during the linear regime of the cosmological density fluctuation evolution. Contrary to the IHR, this second order effect assumes a homogeneous ionisation fraction and relies entirely on the modulation of the Doppler effect by spatial variations of the density field which affect the probability of scattering. Due to its density squared weighting, it peaks at small angular scales, typically arcminute scales in low matter-density flat models, and produces $\mu K \mathrm{rms}$ temperature anisotropies.

To estimate its power spectrum, and therefore its contribution to the temperature anisotropies, we follow the Jaffe \& Kamionkowski (1998) approach. The OV effect can be expressed as the time integral of the velocity field projection along the line of sight modulated by the density field and weighted by the so-called visibility function. The visibility function gives the probability of rescattering of the photons as a function of time (or $z$ ) and encodes all the information relevant for reionisation. The usual approach is to consider that the visibility function follows a Gaussian centred near $z_{\mathrm{i}}$ with a width corresponding to the interval of reionisation

\section{Primary \& Secondary anisotropies@30 GHz}

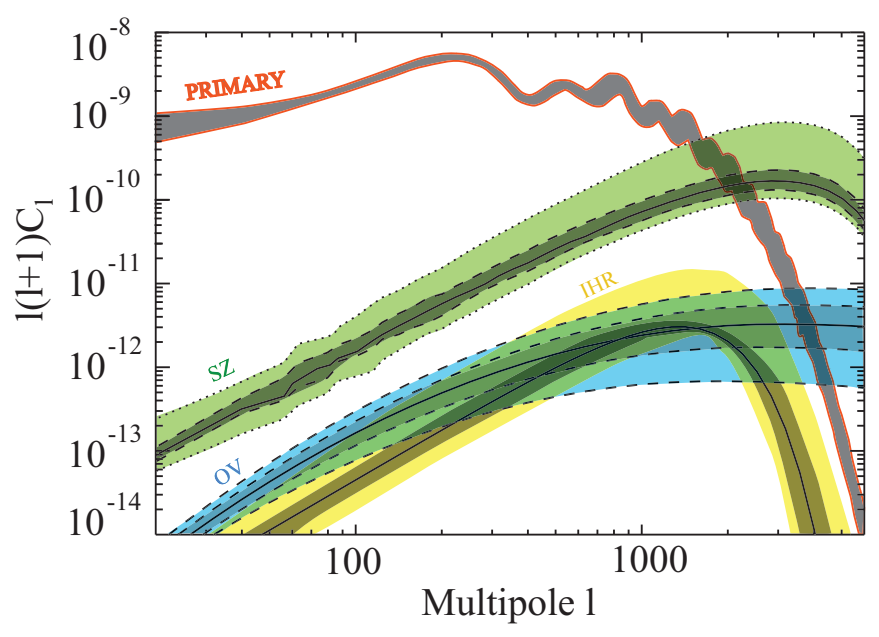

Fig. 2. Primary and secondary CMB anisotropies. The 95\% confidence levels for the primary anisotropies (see text) are plotted together with the $2 \sigma$ envelopes (light shaded regions) and $1 \sigma$ envelopes (heavy shaded regions) for the secondary anistropies (see text). The solid line within the heavy shaded $1 \sigma$ envelope represents, for each contribution, the power spectrum computed for the "concordance model".

$\delta z$. This describes an idealised model for the evolution of the reionisation process. However, cosmological simulations (Gnedin 2000; Gnedin 2001), supported by recent observational data from SLOAN (Becker et al. 2001), indicate a slow first stage of reionisation, called the pre-overlap phase, followed by a fast overlap and post-overlap periods around $z_{\mathrm{i}} \simeq 6$. We thus propose a more realistic model for the visibility function which shape consists of a curve with a smooth growing slope at redshifts higher than $z_{\mathrm{i}}$ and a steep decrease just before $z_{\mathrm{i}}$. This approach increases the amplitude of the OV power spectrum by $50 \%$ at all scales and produces a slight shift of its peak to smaller multipoles (larger angular scales) as compared to previous works. This simple but efficient analytical modeling of the $\mathrm{OV}$ effect will depend on the reionisation redshift, its duration and the cosmological model.

\section{Results}

The main results of our work are plotted in Fig. 2. Constraints on the primary anisotropies are plotted assuming the template of models described in Sect. 2. As we can see, even if multiple oscillations in the CMB data have not been detected at a level better than $2 \sigma$ (see, e.g. Douspis \& Ferreira 2001) the actual data can constrain the region of compatible theoretical models. In particular, the level of primary anisotropies for $\ell>1000$ can be well defined, suggesting that measurements of the power around the first 3 peaks constrain the intrinsic parameters of the model template. Together with the primary anisotropies, we have computed power spectra for the secondary anisotropies. For each contributing signal, we give the power spectra for the so-called "concordance model" $\left(\Omega_{\mathrm{m}}=0.3, \Omega_{\Lambda}=0.7, h=0.71\right)$ (solid line within the heavy shaded region). Taking the values allowed by the CMB analysis (Melchiorri \& Silk 2002) and the HST measurements 
of the Hubble parameter (Freedman et al. 2001), we compute the power spectra of the $2 \sigma$ envelope (with parameters $\omega_{\mathrm{cdm}}=0.06-0.18, h=0.55-0.87$ and $\left.\omega_{\mathrm{b}}=0.018-0.022\right)$, and of the $1 \sigma$ envelope (with parameters $\omega_{\mathrm{cdm}}=0.09-0.15$, $h=0.63-0.79$ and $\left.\omega_{\mathrm{b}}=0.019-0.021\right)$. In Fig. 2, we display both the $2 \sigma$ envelopes (light shaded regions) and $1 \sigma$ envelopes (heavy shaded regions) for the different contributions.

As far as the SZ effect is concerned, we emphasize that the majority of present experiments cannot disentangle it from the primary $\mathrm{CMB}$ due to their observing frequencies. We therefore calculate the TSZ contribution at two frequencies representative of the present experiments: $30 \mathrm{GHz}$ for the radiometric (DASI and CBI-like) experiments and $150 \mathrm{GHz}$ for the bolometric (BOOMERanG, MAXIMA and ARCHEOPS-like) experiments. This component is then added to the KSZ contribution; the total SZ power is then plotted. The SZ power spectrum peaks between $\ell \sim 3200$ and 3600 depending on the cosmological model (but independently of the frequency). Its maximum power, in turn, depends more strongly on the set of cosmological parameters and obviously on the frequency. At $30 \mathrm{GHz}$, the $\ell(\ell+1) C_{\ell}$ for the "concordance model" is $\sim 2 \times 10^{-10}$. At $150 \mathrm{GHz}$, the maximum is about $4 \times 10^{-11}$. We emphasize again that these predictions ought not to be taken at face value, but rather as indicative amplitudes for the $\mathrm{SZ}$ effect. The two other secondary effects induce temperature fluctuations which can be directly expressed in terms of their power spectra regardless of the frequency. The power spectrum of secondary anisotropies generated during the inhomogeneous reionisation peaks in between $\ell \sim 1100$ and 1800 with an amplitude $\sim 3 \times 10^{-12}$ in the "concordance model". As for the OV contribution, the power spectrum peaks around the multipoles $\ell \sim 2000$ and 4000, where the primary anisotropies become subdominant, and its amplitude in the "concordance model" peaks around $\ell \sim 3000$ with amplitude $3 \times 10^{-12}$.

It is worth noting that the SZ anisotropies constitute the dominant secondary contribution when the TSZ effect is not removed, as is the case in the present study. When we compare the power of the primary and secondary anisotropies (Fig. 2), we find that within the $2 \sigma$ envelope, the secondary contribution equals the primary power spectrum between $\ell \sim 1600$ and $\ell \sim 2000$. At $30 \mathrm{GHz}$, the total power spectrum (Fig. 3, upper panel) is already significantly modified beyond the third acoustic peak and the power spectrum is expected to remain almost flat down to $\ell=5000$. At $150 \mathrm{GHz}$, the amplitude of the secondary contributions (dominated by the TSZ effect in our case) is smaller. The observed (total) power spectrum in this case (Fig. 3, lower panel) is noticeably affected only at the position of the sixth acoustic peak. In this case, we do not observe a plateau; the power slowly decreases with increasing $\ell$ s.

\section{Discussion and conclusions}

In this study, we have examined the sub-arcminute structure of the angular power spectrum of the CMB anisotropies. Using a Bayesian approach, we have shown that under the assumption of a wide class of inflationary adiabatic CDM models, the present $\mathrm{CMB}$ data on degree and sub-degree scales can be used to forecast the level of anisotropies. We have therefore
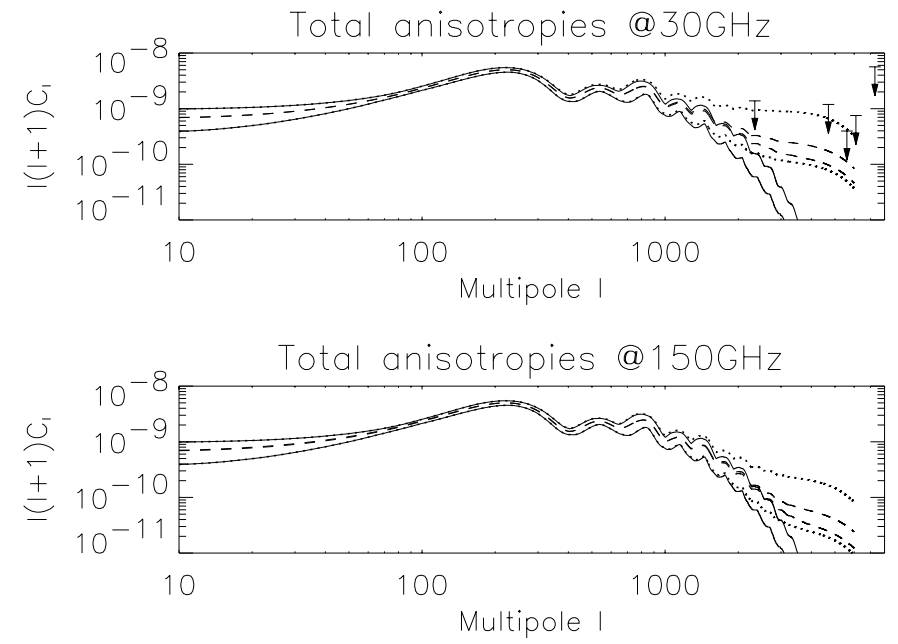

Fig. 3. Total CMB anisotropies. The sum of the primary and secondary anisotropies is plotted: Upper panel, at $30 \mathrm{GHz}$ (for a DASI-like experiment) and lower panel, at $150 \mathrm{GHz}$ (for a BOOMERanG-like experiment). The solid lines represent the predicted primary $\mathrm{CMB}$ region. The dotted and the dashed lines are respectively the 1 and $2 \sigma$ envolpes of the sum (primary + secondary anisotropies). The upper limits (at 95\%) are taken from Springel et al. (2001) and summarise the results from Subrahmanyan et al. (1993), Church et al. (1997), Ganga et al. (1997), Jones et al. (1997), Partridge et al. (1997), Subrahmanyan et al. (1998), Holzapfel et al. (2000).

put strong bounds on the level of contribution from primary CMB anisotropies to the overall spectrum for $\ell>1000$ (on subarcminutes scales). These predictions rely on the experimental results and assume that the present CMB data are free from any unknown foreground contamination including secondary anisotropies.

We have checked that this hypothesis, and the underlying results, remain valid when we generalise the foreground to include the secondary anisotropies. We have therefore computed theoretical predictions for three dominant "candidates" for the secondary anisotropies: SZ effect, OV effect and IHR. Future multi-frequency experiments observing in the millimetre and sub-millimetre will be able to remove most of the TSZ contribution. In this case, the remaining SZ signal will be associated with the KSZ effect. It is one order of magnitude lower than the values quoted in Fig. 2, and has the same spectral signature as the primary anisotropies. The remaining SZ contribution will therefore be larger than, or of the same order of, the OV and IHR contributions (which are at the same level). Since all three signals are spectrally confused, the multi-frequency experiments will measure the sum of the three contributions. The resulting signal is rather small; it is thus not expected to significantly alter the primary anisotropies. However nowadays, the present status of the CMB experiments (specifically, their frequency coverage) does not allow us to remove a possible contribution from the TSZ effect of a cluster population. In this context, the secondary contribution to the CMB power spectrum is dominated by TSZ.

We have shown that such a contribution can be large enough to significantly modify the CMB power spectrum 
beyond the third acoustic peak. This alteration is more pronounced at $30 \mathrm{GHz}$ than at $150 \mathrm{GHz}$, which could be the case if the errors bars for the $30 \mathrm{GHz}$-experiments are found to be larger than for the $150 \mathrm{GHz}$-experiments. The predicted $2 \sigma$ envelope for the total power spectrum (Fig. 3, upper panel, dotted lines) is displayed together with the upper limits $(95 \%)$ obtained from small scale experiments quoted in Springel et al. (2001) (Subrahmanyan et al. 1993; Church et al. 1997; Ganga et al. 1997; Jones et al. 1997; Partridge et al. 1997; Subrahmanyan et al. 1998; Holzapfel et al. 2000). We note that the upper limits are close to our $2 \sigma$ region defined by the cosmological parameters allowed by the actual constrains. The good consistency between BOOMERanG and DASI data (at 150 and $30 \mathrm{GHz}$ respectively) together with the upper limits at small scales indicates that the present $C M B$ data already constrain rather well the contribution from the secondary effects, TSZ effect in particular. However, this contribution is highly dependent on the cluster abundance and their internal physics which can modify our predictions. In addition, this result has been obtained under a theoretical prior. The possible presence of "non-standard" mechanisms like primordial voids (Sakai et al. 1999; Cooray 2002; Griffiths et al. 2002), bumps (Griffiths et al. 2001), scale dependence of the primordial spectral index (Kosowsky \& Turner 1995) or topological defects (Avelino \& Martins 2000), for example, can significantly change our conclusions. Fortunately, these mechanisms can be distinguished in principle from the models reported here by combination with different datasets such as those from deep redshift galaxy surveys, for example.

Future small-scale CMB data such as that expected from CBI (Mason et al. 2002), ARCHEOPS (Amblard et al. 2001; Benoit et al. 2001), will be helpful for refining our results. Ultimately, MAP and Planck satellites will provide us with much more accurate measurements. Barring the degeneracies, these experiments will allow a much smaller range of possible cosmological models. At that time, the measurements of the $\mathrm{CMB}$ anisotropies after the third peak will not only constrain the cosmological model through parameter estimation, but will also unable us to probe, via the secondary anisotropies (e.g. $\mathrm{SZ}$ ), the formation and evolution of structures ${ }^{1}$.

Acknowledgements. This work was partially supported by the European TMR CMBnet. PGC is supported by the Fundação para a Ciêcia e a Tecnologia. NA thanks T.F. and the Denys Wilkinson Building for "hospitality". The authors thank M. Douspis and P. Ferreira for helpful discussion, and M. Kunz for his comments. We also thank an anonymous referee for the comments and suggestions that helped in improving the paper.

\section{References}

Aghanim, N., Désert, F. X., Puget, J. L., \& Gispert, R. 1996, A\&A, 311,1

Aghanim, N., Górski, K. M., \& Puget, J. L. 2001, A\&A, 374, 1

Albrecht, A., Coulson, D., Ferreira, P. G., \& Magueijo, J. 1996, Phys. Rev. Lett., 76, 1413

1 When we were about to submit this article, a similar study came out (Cooray 2002) in which the author examined the contributions from secondaries including IWS and weak lensing for the fiducial $\Lambda \mathrm{CDM}$ model.
Amblard, A. 2001, for the ARCHEOPS collaboration [astro-ph/0112205]

Avelino, P., \& Martins, C. 2000, Phys. Rev. Lett., 85, 1370

Becker, R. H., et al. 2001, AJ, 122, 2850

Benoit, A., et al. 2001 [astro-ph/0106152]

Benson, A. J., Nusser, A., Sugiyama, N., \& Lacey, C. G. 2001, MNRAS, 320, 153

Birkinshaw, M., \& Gull, S. F. 1983, Nature, 302, 315

Birkinshaw, M. 1999, Phys. Rep., 310, 97

Bouchet, F. R., \& Gispert, R. 1999, New Astron., 4, 443

Bridle, S. L., Crittenden, R., Melchiorri, A., et al. 2001 [astro-ph/0112114]

Bruscoli, M., Ferrara, A., \& Scannapieco, E. 2002, MNRAS, 330, L43

Church, S. E., et al. 1997, ApJ, 484, 523

Ciardi, B., Ferrara, A., Marri, S., \& Raimondo, G. 2001, MNRAS, 324,381

Cooray, A. 2000, Phys. Rev. D, 62, 3506

Cooray, A. 2002, in 2001 Coral Gables conference on cosmology and particle physics, ed. B. Kursunoglu, \& A. Perlmutter, American Institute of Physics Conference Proceedings [astro-ph/020304]

Cooray, A., \& Melchiorri, A. 2002 [astro-ph/0204250]

da Silva, A. C., Barbosa, D., Liddle, A. R., Thomas, P. A. 2000, MNRAS, 317, 37

de Bernardis, P., et al. 2000, Nature, 404, 955

de Bernardis, P., et al. 2002, ApJ, 564, 559

Dodelson, S., \& Jubas, J. M. 1995, ApJ, 439, 503

Dodelson, S., \& Knox, L. 2000, Phys. Rev. Lett., 84, 3523

Douspis, M., \& Ferreira, P. G. 2001 [astro-ph/0111400]

Durrer, R., Novosyadlyj, B., \& Apunevych, S. 2001 [astro-ph/0111594]

Freedman, W. L., et al. 2001, ApJ, 553, 47

Ganga, K., et al. 1997, ApJ, 484, 517

Gnedin, N. Y. 2000, ApJ, 535, 530

Gnedin, N. Y. 2001 [astro-ph/0110290]

Gnedin, N. Y., \& Jaffe, A. H. 2001, ApJ, 551, 3

Griffiths, L. M., Silk, J., \& Zaroubi, S. 2001, MNRAS, 324, 712

Griffiths, L. M., Kunz, M., \& Silk, J. 2002 [astro-ph/0204100]

Gruzinov, A., \& Hu, W. 1998, ApJ, 508, 435

Gunn, J. E., \& Peterson, B. A. 1965, ApJ, 142, 1633

Halverson, W., et al. 2001 [astro-ph/0104489]

Hanany, S., et al. 2000 [astro-ph/0005123]

Hobson, M. P., Jones, A. W., Lasenby, A. N., \& Bouchet, F. R. 1998, MNRAS, 300, 1

Holzapfel, W. L., et al. 1998, ApJ, 539, 57

Hu, W., Scott, D., \& Silk, J. 1994, Phys. Rev. D, 49, 648

Hu, W., \& Dodelson, S. 2002, ARA\&A [astro-ph/0110414]

Jaffe, A. H., \& Kamionkowski, M. 1998, Phys. Rev. D, 58, 043001

Jones, M. E., et al. 1997, ApJ, 479, L1

Knox, L., Scoccimarro, R., \& Dodelson, S. 1998 [astro-ph/9805012]

Komatsu, E., Kitayama, T., Refregier, A., Spergel, D. N., \& pen, U. L. 2000, Marcel Grossmann meeting [astro-ph/0012196]

Kosowsky, A., \& Turner, M. S. 1995, Phys. Rev. D, 52, 1739

Lee, A. T., Ade, P., Balbi, A., et al. 2001, ApJ, 561, L1

Majumdar, S. 2001, ApJ, 555, L7

Mason, B., and the CBI collaboration 2002, 199th AAS meeting

Mauskopf, P. D., et al. 2000, ApJ, 536, L59

Melchiorri, A., et al. 2000, ApJ, 536, L63

Melchiorri, A., \& Silk, J. 2002, in preparation

Miller, A. D., et al. 1999, ApJ, 524, L1

Miller, C. J., Nichol, R. C., Genovese, C., \& Wasserman, L. 2001 [astro-ph/0112049]

Netterfield, C. B., et al. 2001 [astro-ph/0104460] 
Ostriker, J. P., \& Vishniac, E. T. 1986, ApJ, 306, L51

Partridge, R. B., Bruce, R. E. A., Fomalont, E. B., Kellermann, K. L., \& Windhorst, R. A. 1997, ApJ, 483, 38

Peebles, P. J. E. 1993, in Principles of physical cosmology (Princeton University Press)

Press, W. H., \& Schechter, P. 1974, ApJ, 187, 425

Pryke, C., Halverson, N. W., Leitch, E. M., et al. 2001 [astro-ph/0104490]

Rees, M. J., \& Sciama, D. W. 1986, Nature, 511, 611

Refregier, A., \& Teyssier, R. 2000 [astro-ph/0012086]

Rephaeli, Y. 1995, ARA\&A, 33, 541

Sakai, N., Sugiyama, N., \& Yokoyama, J. 1999, ApJ, 510, 1

Seljak, U. 1996, ApJ, 463, 1

Sheth, R. K., \& Tormen, G. 1999, MNRAS, 308, 119

Snoussi, H., Patanchon, J. F., Macias-Perez, J. F., Mohammed-Djafari, A., \& Delabrouille, J. 2001 [astro-ph/0109123]
Springel, V., White, M., \& Hernquist, L. 2001, ApJ, 549, 681

Stompor, R., Abroe, M., Ade, P., et al. 2001, ApJ, 561, L7

Subrahmanyan, R., Ekers, R. D., Sinclair, M., \& Silk, J. 1993, MNRAS, 263, 413

Subrahmanyan, R., Kesteven, M. J., Ekers, R. D., Sinclair, M., \& Silk, J. 1998, MNRAS, 298, 1189

Sunyaev, R. A., \& Zel'dovich, Ya. B. 1980, ARA\&A, 18, 537

Viana, P. T. P., \& Liddle, A. R. 1999, MNRAS, 303, 535

Vishniac, E. T. 1987, ApJ, 322, 597

Wang, X., Tegmark, M., \& Zaldarriaga, M. 2001 [astro-ph/0105091]

White, M., \& Cohn, J. D. 2002 [astro-ph/0203120]

Wu, J. H. P. 2000 [astro-ph/0012207] 